\title{
Underwater energy harvesting system based on plucked-driven piezoelectrics
}

\author{
Daniel M. Toma; Joaquin del Rio; Montserrat Carbonell-Ventura; Jaume Miquel \\ Masalles; \\ SARTI Research Group. Electronics Dept. Universitat Politècnica de Catalunya (UPC). \\ Rambla Exposició 24, 08800, Vilanova i la Geltrú. Barcelona. Spain. +(34) 938967 \\ 200 \\ daniel.mihai.toma@upc.edu
}

Today, both the underwater communication technologies and the sensor technology are mature enough to motivate the idea of underwater sensor networks. Underwater wireless sensing systems are envisioned for stand-alone applications and as an addition to cabled systems. For example, cabled ocean observatories are being built on submarine cables to deploy an extensive Fiber-optic network of sensors (cameras, wave sensors and seismometers) covering miles of ocean floor. These cables can support communication access points, very much as cellular base stations are connected to the telephone network, allowing users to move and communicate from places where cables cannot reach. In Figure 1 is illustrated the OBSEA underwater observatory having fiber-optic and power cable for communication with the shore station and acoustic modem for communication with underwater wireless instruments.

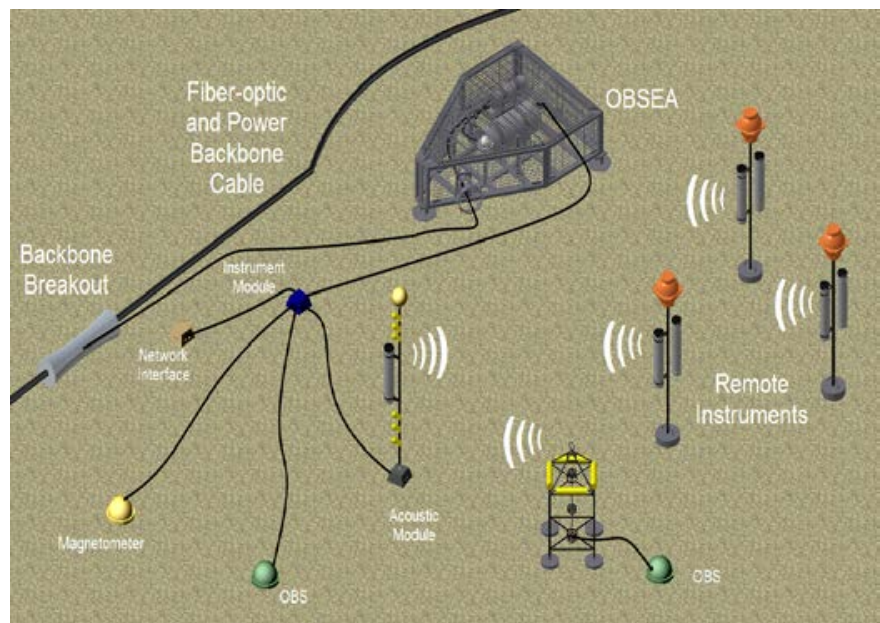

Figure 1 Diagram of underwater observatory. Shows hybrid communication system, measurement equipment with wireless and wired access in underwater environment

However, to turn this idea into reality, one must face the problem of providing powering solutions for long term deployments. The energy requirements of low-power electronics have steadily decreased with advancements in efficient circuitry such that energy harvesting systems can be considered feasible solutions in providing power to self-powered systems. Among the ocean areas where harvestable energy is available, the most interesting ones are the areas with tidal and ocean currents and the shallow water areas with significantly wave-induced oscillatory currents at the sea bottom. Particular, wave-induced oscillatory currents at the sea bottom are interesting for harvesting device because of the body dynamics placed in these areas. The path of water displacement in gravity waves is roughly circular motion. In deep water, the path becoming smaller with depth before finally disappearing as the depth increases beyond the zone of wave disturbance and wave motion does not reach the seabed. In shallow water, the water particles have an elliptical orbit and wave motion is felt at the seabed.

In this paper, we present the development of a new harvesting system for underwater energy harvesting. New system configuration and piezoelectric bimorph materials are employed to improve the efficiency of energy conversion. In contrast with the conventional bimorph, resonant, vibrational energy harvester, the plucked-driven piezoelectric harvester shown in Figure 2 comprises two beams. One is a piezoelectric generating beam with a high resonant frequency; the second is a short driving beam, plectra. When the plectra impacts the generating beam, vibration is excited first at the system's coupled vibration frequency and then at the generating beam's higher resonant frequency, producing electrical power. 


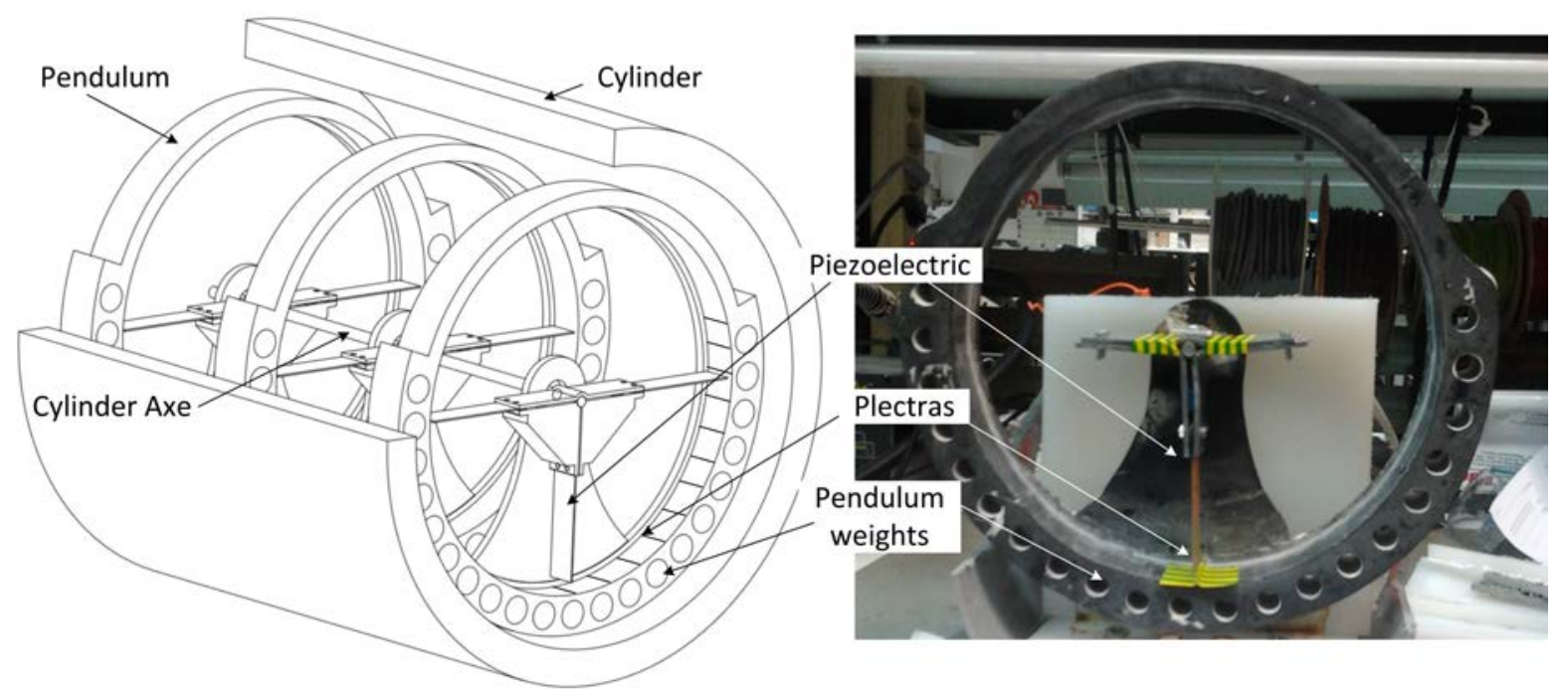

Figure 2 Schematic representation of the proposed underwater energy harvesting systems

The underwater harvesting device designed to use this planked-driven approach is based on an internal pendulum, with plectra's attached to the internal radius and piezoelectric beams fixed to the pendulum axe. The underwater harvesting device was demonstrating using a $25.2 \mathrm{~cm}$ external diameter, 20.2 internal diameter, $2.5 \mathrm{~cm}$ thick epoxy resin pendulum with a mass of $330 \mathrm{~g}$. On the internal diameter of the pendulum there where attached vinyl plectra's of $5.7 \mathrm{~mm}$ long, $20 \mathrm{~mm}$ wide, $0.3 \mathrm{~mm}$ thick. Three piezoelectric beams, model V21BL (Mide Technology Corporation) of $9.05 \mathrm{~cm}$ long, $1.44 \mathrm{~cm}$ wide and $0.78 \mathrm{~mm}$ thick where attached to the pendulum axe with a offset angle of 90 degrees.

Figure 3 plots the instantaneous output voltage of a single plunked vibration piezoelectric beams for a driving rotation of the harvesting device of $25 \mathrm{rad} / \mathrm{s}$ at $0.33 \mathrm{~Hz}$ and with several plectra's facing the piezoelectric beam and with $5 \mathrm{~mm}$ gap. The maximum peak-to-peak voltage is $30 \mathrm{~V}$. The impact phase between the piezoelectric beam and the plectra's appears several times per cycle depending on the maximum rotation angle of the harvesting device and after each impact occurs the free vibration phase. Experimental results shows that the proposed energy harvester achieves a maximum power density of $350 \mu \mathrm{W} / \mathrm{cm} 3$.

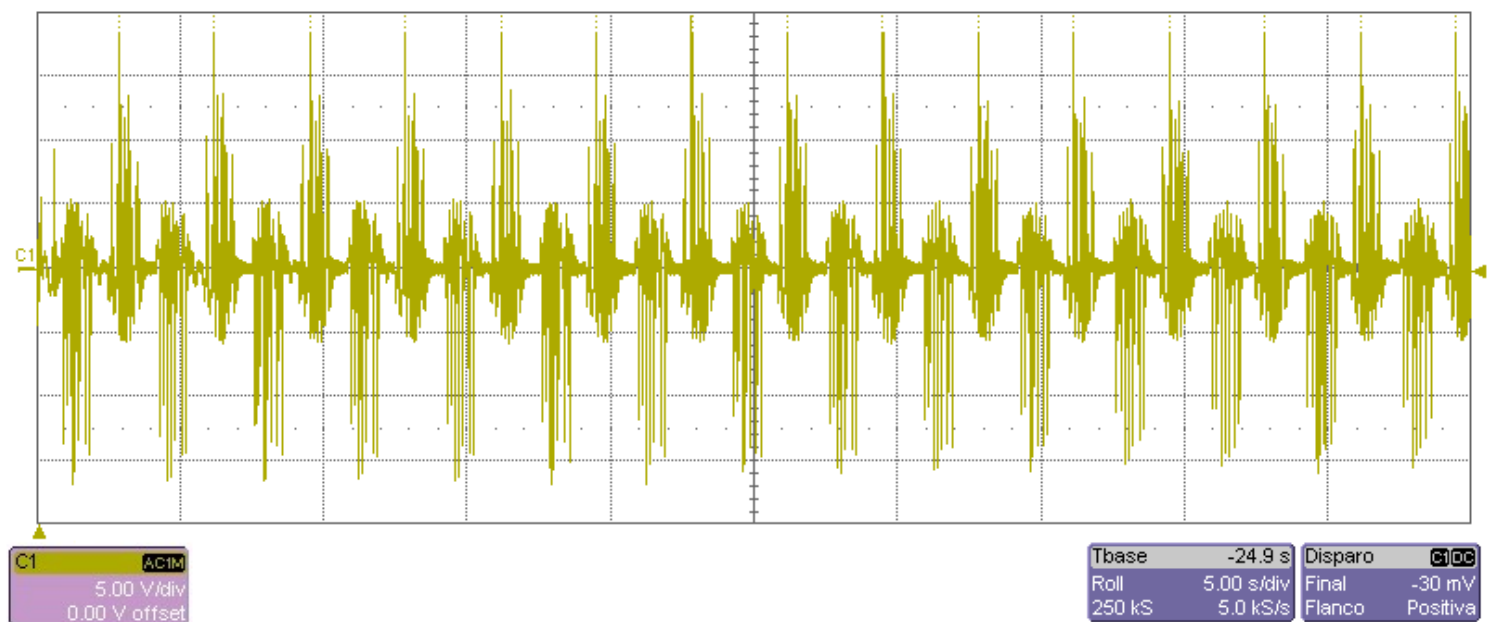

Figure 3 Output voltage of a single piezoelctric plotted versus time for a rotation of the harvesting device of $25 \mathrm{rad} / \mathrm{s}$ at $0.33 \mathrm{~Hz}$ and with several plectra with $5 \mathrm{~mm}$ gap facing the piezoelectric beam. 